\title{
Construction and Analysis of Light Energy Transfer Model Based on Underwater Environment
}

\author{
Liu Yan ${ }^{1, a}, Y u L^{2, b}$ \\ ${ }^{1,2}$ PLA 91550 Unit, Dalian,116023, China \\ aemail:liuyan8721@sina.com, bemail:yulu1503619387@163.com
}

\section{Keywords: Underwater imaging; Energy transfer; Optical transmission characteristics}

\begin{abstract}
The application prospect of underwater imaging detection is wide in the fields of military operations, marine development and engineering applications. Considering the complex marine environment, the energy transfer process of underwater imaging sensor is analyzed on the basis of optical seawater transmission characteristics. The relationship between photon energy received by underwater imaging receiver, environment, target and auxiliary light source is studied, and it provides technical support for the construction of underwater imaging system.
\end{abstract}

\section{Introduction}

Ocean is a complex physical, chemical and bio-optical system. The underwater light is strongly scattered and absorbed because of the sea water non-uniformity[1]. For the application prospect of underwater imaging detection is wide in the fields of military operations, marine development and engineering applications, the underwater imaging detection is highly regarded by every national military. Underwater imaging detection system can clearly record and image the underwater sudden incident under the faint light and high scattering medium environment. Underwater imaging detection system is mainly composed of imaging devices, auxiliary lighting and control recorder. Due to the impact of seawater characteristics, it is not that under the water the receiving device can get more energy with the greater intensity of auxiliary illumination[2]. Therefore, underwater light energy model is established to design auxiliary light source and camera parameters, lay light source device and camera rationally by target characteristics. The relationship between intensity of auxiliary illumination, light source intensity, divergence angle, target distance and receiver energy is studied, which provides technical support for the selection of test sea area, the design of underwater auxiliary light source, the location of light source, the analysis of minimum imaging distance and device selection.

\section{Procedure of underwater optical energy transfer}

Underwater imaging detection system is mainly composed of imaging devices, auxiliary lighting and control recorder. In the underwater detection system, the layout of underwater light source, target and receiver is shown in Fig. 1.

As shown in Fig.1, $L$ is the distance between light source and target; $r$ is the distance between receiver and target; $S_{0}$ is the distance between light source and receiver, which is defined as optic axis distance. The intensity of light source is denoted by the function $I(\theta)$.

The seawater light attenuation is caused by the seawater absorption and scattering of light. The decay of light energy in seawater is exponentially decayed by light attenuation coefficient. On the one hand, the energy of the underwater light field is weakening due to the absorption effect, but the absorption does not affect the distribution of the light field formed by the light; on the other hand, the scattering direction changes the photon to form a new underwater light field distribution, which is the diffuse light field. 


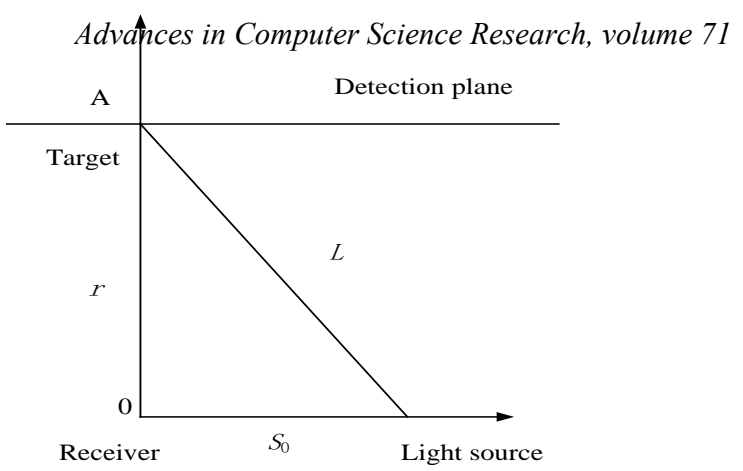

Fig.1 Layout of underwater light source, target and receiver

When light transmits in the underwater, the light of information received by receiver consists of two parts: direct beam, which is reflected from the target and absorbed by the aqueous medium and suffers scattering loss, and the aqueous medium scattered light beam between light source and target. The scattered beam is divided into forward scattered light and back scattered light. The direct light portion is most sensitive to the distance between the imaging system height and the illumination system. The forward scattered light affects the resolution of the target detail, and the change of the backscattered light is the least sensitive, but with the increase of the measuring distance, the contrast of the imaging is reduced[3]. In order to improve the imaging quality of underwater imaging, general underwater imaging systems have underwater auxiliary lighting system. Affected by the attenuation mechanism of the sea light, the intensity of auxiliary illumination is not the bigger the better. The light backscattering increases with improving illumination, the imaging is not effective.

\section{Establishment of underwater optical energy transfer model}

In the underwater light field, the light irradiated on the target consists of the natural light and auxiliary lighting, which is received by the receiver after the target reflection. The energy of direct light and scattered light is transferred to the imaging device receiver, and then the underwater natural light is absorbed and scattered by aqueous medium. The auxiliary light from auxiliary illumination equipment is absorbed and scattered by the water medium, then the direct light and scattered light energy is transferred to the target. This light is absorbed and reflected by the target, and absorbed and scattered by water again, then the direct light and scattered light energy is transferred to the imaging device receiver.

\section{Light energy transfer model of object}

The light energy received by object consists of natural light and auxiliary light. Natural light ultimately reaches the measured target through a certain depth of transmission underwater. After auxiliary illumination light transmit through a certain distance of the transmission of water medium, the light energy incepted by target is the energy of direct light and scattered light from the auxiliary light source.

\section{Natural light energy transfer model of object}

Natural light mainly includes sunlight and daylight[4]. In the seawater, blue-green light in natural light is called the "underwater window" and can be transmitted to the water with less attenuation. The change of sun's illumination received by ground is great on different weather conditions. Generally, in order to get enough natural light underwater illumination, good weather conditions and the sun height are selected. While natural light transfers into the water through the water medium, the effective spectral energy is attenuated quickly with the water depth. From the spectral distribution curve of sunlight, we can get that the light whose wavelength is longer than $0.6 \mu \mathrm{m}$ is absorbed seriously by seawater, and once the water depth is more than $10 \mathrm{~m}$, there is only the blue-green light. We know that blue-green light accounts for about $40 \%$ of visible light band[5]. The blue-green light illumination of different depths can be expressed as follows

$$
E=E_{0} e^{-c z}
$$

Where $E$ is underwater illumination, $E_{0}$ is the offshore light intensity, $c$ is the transmission 
attenuation coefficients of the different wavelengths, and $\mathrm{z}$ is the depth of water, and generally $E_{0}=5 \times 10^{3}$ lux .

\section{Direct light transfer model of object}

From the layout of underwater light source, target and receiver are shown in Fig.1. Using collimated light beam, according to Lambert Bell attenuation rule of the aqueous medium and illuminance square reciprocity law, the Illumination generated by the direct light of area element $d s$ at target $A$ can be expressed as follows

Where $I(\theta)$ is the radiator source light intensity and $c$ is the volume attenuation coefficient of aqueous medium.

\section{Scattering light transfer model of object}

In practical engineering applications, the illumination light source has a certain divergence angle, so that part of the scattered photons generated by the arbitrary divergence angle will enter the other divergence angle direction within the beam range. Therefore, the light intensity on the direction angle is compensated or enhancement. It has been shown that the illumination intensity of the long-distance target can be enhanced to some extent due to the effective illumination provided by the scattered light energy[6].

Duntley calculated the energy of the scattering light which can provide efficient lighting at the distance $L$ from the light source, which overcomed the error caused by the above object illuminance estimation using only direct light component. It is expressed as follows

where $\varphi$ is the whole opening angle of the radiation beam source; $k$ is the attenuation coefficient of diffuse light volume, and on the condition of the general water quality, $c / k=2.7$ is a more generic conversion relationship; $I$ is the intensity of light source; $L$ is the distance between the light source and the target object.

\section{Total illumination model of object}

According to the Underwater vision system as shown in Fig.1, the total illumination energy received by target object consists of the direct and the diffuse light energy from natural light, the direct light energy from light source and the scattered light energy from light source, namely:

(4)

\section{Energy transfer model of the return light}

The return light energy received by the receiver includes the return direct light energy and the return scattered light energy. Underwater target is generally Lambertian diffuse reflector, and the received light energy of the target object is reflected by the target, and ultimately reaches the CCD detector after a certain range of transmission.

\section{Direct illuminance model of the return light}

The illuminated object is assumed to be a Lambertian diffusion reflector, and the target reflectance is expressed as $\rho$. After reflection of the light energy, the object can be regarded as a Lambertian light source having an area $S$ which has the same brightness distribution in all directions. The luminance distribution on the object can be expressed as follows:

(5)

where $\rho$ is the reflectivity of the target object; $L T$ is the luminance distribution on the object; $E_{\text {Tsum }}$ is the total target illuminance.

As shown in Fig.1, if illuminated area of the object is $S$, the receiver distance $r$, and the flux of the light transferring through the distance $r$ and reaching the outside of receiver under the effect of the aqueous medium attenuation coefficient $c$ is $\tau$. The lens transmission coefficient of the optical imaging system is $\tau$, which is commonly used underwater infinity focus, fixed focal length 
lens system of the CCD. The illuminance value of direct light received by the CCD imager from the target is given by

Where $D$ is the receiver aperture size, $\pi D^{2} / 4$ is the receiver light area, and $\mathrm{f}$ is the focal length of camera.

Taking Eq. (4) into Eq. (6), we can get that

Eq. (7) is the direct light illuminance value formula calculated on the CCD imaging plane according to the method of the underwater auxiliary illumination geometry shown in Fig.1.

It can be seen that the light intensity value received by the receiver is proportional to the light intensity $I(\theta)$, the object reflection coefficient $\rho$, and the transmittance of the optical system of the imaging lens $\tau$; the photon energy from the light source finished $e^{-c(r+l)}$ of e exponential decay, which returns to the receiver through object reflection; $D / f$ is the lens relative aperture of the receiver, which can be found directly or indirectly in the general parameters of the imaging device.

\section{Received backscattered light illumination model}

As the scattered light component provides efficient illumination in the process of light energy transport from light source to target, the light is reflected by the target object who having reflected Lambert diffuse reflection characteristics. The illuminated target object, which is at a distance $\mathrm{r}$ of the receiver and whose area is $S$, is considered to be the Lambertian light source whose brightness is $L_{T}$. The radiation intensity $I_{T}(\theta)$ in all directions can be expressed as:

Where $\theta$ is the angle between the direction of light radiation intensity and the normal direction of target area element $d s ; S$ is the area of the illuminated object.

In the actual calculation, we assume that the normal direction of the target area element $d s$ coincides with the based spindle of the receiver perspective, and $\theta$ is $0^{\circ}$. Lambert target light source has a diffuse reflection characteristics, light divergence angle is taken as $\pi$, and optical lenses permeation coefficient in the imaging systems is $\tau$. When the light from Lambert light source arrived at the receiver at the effect of aqueous medium attenuation, the effective illuminance value provided by scattered photons at the receiver can be expressed as:

9)

Eq. (9) is the scattered light illuminance value formula calculated on the CCD imaging plane according to the method of the underwater auxiliary illumination geometry shown in Fig.1.

It can be seen that the light intensity value received by the receiver is proportional to the light intensity $I(\theta)$, the object reflection coefficient $\rho$, and the transmittance of the optical system of the imaging lens $\tau$; the photon energy from the light source finished $e^{-c(r+l)}$ of e exponential decay, which returns to the receiver through object reflection; $D / f$ is the lens relative aperture of the receiver, which can be found directly or indirectly in the general parameters of the imaging device.

\section{The target total light illumination of receiver}

In the underwater detection system, the photon energy received by the illumination object is derived from the irradiation of the natural light and the illumination of the auxiliary illumination source. CCD imaging photoelectric cells can be effectively triggered or not, depending on the target reflection scattered light and direct light illumination on the CCD image surface. The total intensity of illumination reflected from the target object back to a receiver, includes direct illuminance component $E_{R d}$ and back scattering component $E_{R s}$, so the total intensity value of illumination can be expressed as: 
Where $\varphi$ is the whole opening angle of the radiation beam source in radians; $k$ is the attenuation coefficient of diffuse light volume, and on the condition of the general water quality, $c / k=2.7$ is a more generic conversion relationship; $I$ is the intensity of light source; $L$ is the distance between the light source and the target object; $r$ is the distance between the receiver and the target object, $D / f$ is the relative aperture of the receiver, $p$ is the reflection coefficient, $\tau$ is the optical Imaging system transmittance.

\section{Simulation and Analysis}

Eq. (10) provides an overall illuminance calculation model that transmits light energy to the receiver for the underwater imaging system. The light energy model received provides a selection from the technical support such as the selected test waters, underwater auxiliary light source design, layout position of the light source and the minimum imaging analysis. By substitute the water parameters, equipment optical parameters, equipment laying geometrical parameters into Eq. (10), the effective size of the illumination $E_{\text {Rsum }}$ on the receiving device imaging photoelectric cells can be calculated. The common underwater imaging CCD device has its own minimum illuminance sensitivity index. For example, a camera sensitivity ISO is 2000, the frequency of the selected shooting is $500 \mathrm{fps}$, the minimum illumination of the calculation camera is 0.2 lux. When the device is used as underwater imaging receiver with $E_{R s u m}<0.2$ lux, the energy of receiving photon is insufficient to trigger the photosensitive unit of CCD, and effective imaging is not formed[8]. Tab.1 is the underwater imaging system simulation parameter setting table. The total illuminances received by receiver were got using MATLAB software, which are shown in Fig.2 to Fig.6.

The simulation results show that:

a. Underwater imaging systems can improve the receiver's illumination with the help of natural light and auxiliary lighting.

b. From Fig. 3 and Fig. 5, we can see that different water depth and different attenuation coefficient of sea water have an influence on the receiver illumination, and the deeper the depth, the greater the attenuation coefficient of the sea water, the smaller the illuminance of the receiver. The result indicates that the influence of seawater environmental conditions on underwater imaging is very important. 
Table 1 Simulation parameter setting table of underwater imaging system

\begin{tabular}{|c|c|c|c|c|c|}
\hline $\begin{array}{c}\text { Paramete } \\
\text { r name }\end{array}$ & $\begin{array}{c}\text { Water surface } \\
\text { illumination(lu } \\
\text { x) }\end{array}$ & $\begin{array}{c}\text { Working } \\
\text { depth(m) }\end{array}$ & $\begin{array}{c}\text { Light- } \\
\text { emitting } \\
\text { intensity (cd) }\end{array}$ & $\begin{array}{c}\text { Full-angle } \\
\text { light } \\
\text { source(rad) }\end{array}$ & $\begin{array}{c}\text { Aqueous medium } \\
\text { attenuation } \\
\left.\text { coefficient( }{ }^{-1}\right)\end{array}$ \\
\hline $\begin{array}{c}\text { Paramete } \\
\text { r value }\end{array}$ & $5 \times 10^{3}$ & $10 \sim 30$ & $(0 \sim 3) \times 10^{5}$ & $0.15 \sim 1.5$ & $0 \sim 0.25 \mathrm{~m}^{-1}$ \\
\hline $\begin{array}{c}\text { Paramete } \\
\text { r name }\end{array}$ & $\begin{array}{c}\text { Light } \\
\text { Wheelbase (m) }\end{array}$ & $\begin{array}{l}\text { Distance } \\
\text { between } \\
\text { receiver } \\
\text { and } \\
\text { target(m) }\end{array}$ & $\begin{array}{c}\text { Reflection } \\
\text { coefficient of } \\
\text { target }\end{array}$ & $\begin{array}{c}\text { Optical } \\
\text { imaging } \\
\text { system } \\
\text { transmissivity }\end{array}$ & $\begin{array}{c}\text { Relative aperture of } \\
\text { the receiver }\end{array}$ \\
\hline $\begin{array}{c}\text { Paramete } \\
\text { r value }\end{array}$ & $0.3 \sim 1.5$ & $5 \sim 20$ & 0.4 & 0.9 & $1 / 1.4$ \\
\hline
\end{tabular}

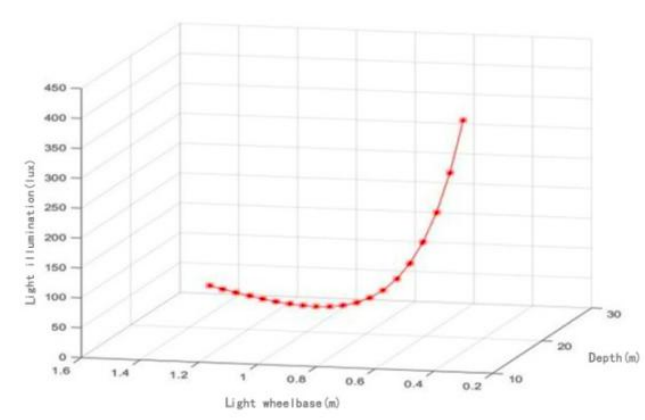

Fig. 2 Depth, light wheelbase, total illuminance illuminance

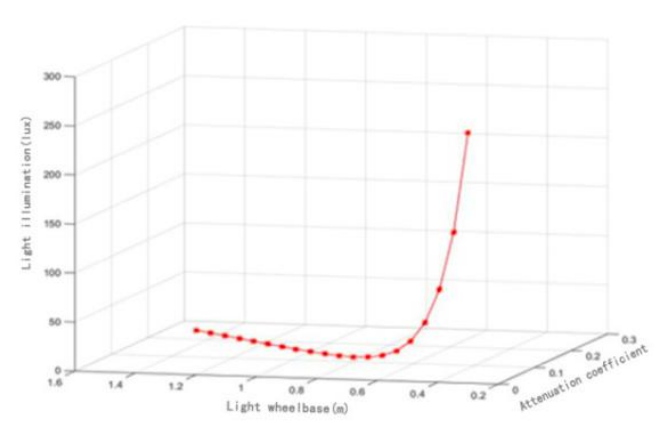

Fig. 4 Light wheelbase, attenuation coefficient, coefficient,

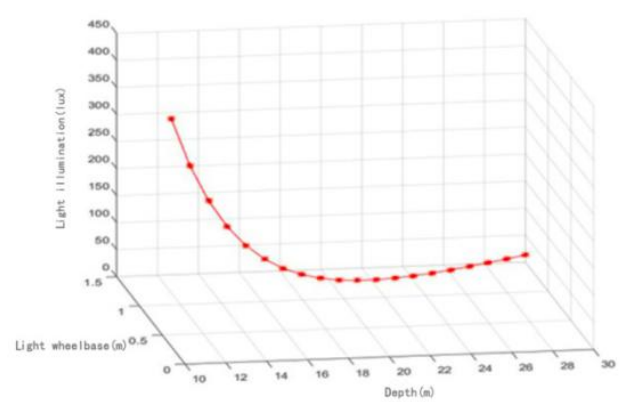

Fig. 3 Depth, light wheelbase, light

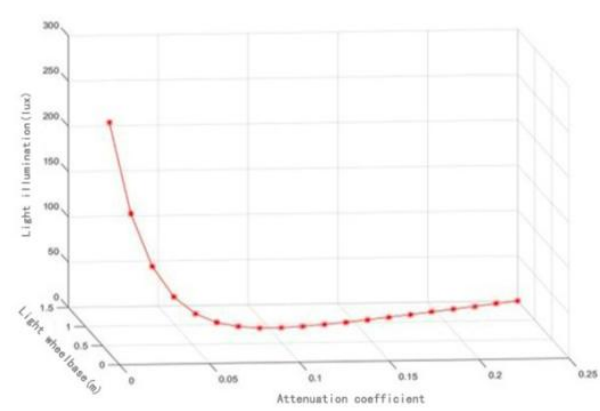

Fig. 5 Light wheelbase, attenuation light illuminance 
c. It can be seen from Fig. 6 that different divergence angle and different luminous intensity have an impact on illumination of the receiver, and the smaller the divergence angle, the greater the luminous intensity, the greater the receiver receiver illumination. It shows that the design of auxiliary illumination light source has a great influence on underwater imaging.

d. Fig. 2 and Fig. 4 show that the different optical axis have an impact on illumination of the receiver, and the smaller the optical axis, the greater the receiver receiver illumination. It is illustrated that the arrangement of underwater auxiliary illumination field directly affects the receiving illumination, and reasonable arrangement of underwater light field is an effective method to obtain underwater clear water image.

\section{Conclusion}

Imaging technology is the main measure to obtain the underwater motion attitude of missile. In the underwater light field, the light irradiated on the target includes the natural light and auxiliary lighting. The light energy received by receiver mainly includes the direct beam and scattered beam which are reflected by the target. The energy requirement of the target information received by the receiver is a necessary condition for the underwater imaging system to obtain a clear and reliable image. The optical energy transfer process of underwater imaging system has been analyzed, and the relationship between the auxiliary illuminance, light source intensity, divergence angle, distance of target and receiver energy has been studied, which provides technical support for selection of test sea fields, design of the underwater auxiliary light source, layout of light source, analysis of the minimum imaging distance and selection of detection devices.

\section{References}

[1] HE Zhao-cai, Hu Bao-an. The Light Measurement System[M]. National Defense Industry Press, 2002.

[2] XIANG Shi-ming,NI Guang-qiang,et al.The Principle of Photo electronic Imaging Devices[M].Beijing: National Defense Industry Press, 1999.

[3] WU Zheng,SUN Ao,et al. Test and Measurement-control Technique for Sea Range [M]. National Defense Industry Press, 2007.

[4] Xu Hongmei, Research on Target Detection of Underwater Non-uniform Light Field, Doctoral Dissertation of Ocean University of China, 2009

[5] Liu Yan, Performance evaluation of underwater low light imaging system based on energy transfer chain [J] Journal of Projectiles, Rockets and Guidance, 2010(5):196-198.

[6] SUN Chuan-Dong, Optical properties of water and its effect on underwater imaging [J]. Journal of Applied Optics 2000,21 (4): 39-46.

[7] Zhang Yujin, Image Engineering (Part 2) Image Comprehension [M], Beijing: Tsinghua University Press, February 2007 Second Edition:63-64. 